\title{
MRI-Based Neuroanatomical Predictors of Dysphagia, Dysarthria, and Aphasia in Patients with First Acute Ischemic Stroke
}

\author{
Heather L. Flowers ${ }^{a}, b, f \quad$ Mohammed A. AlHarbic $^{c}$ David Mikulis ${ }^{d}$ \\ Frank L. Silver ${ }^{d, e} \quad$ Elizabeth Rochon ${ }^{f, g}$ David Streiner ${ }^{h, i}$ \\ Rosemary Martino ${ }^{f, j}$ \\ a Department of Rehabilitation Sciences, University of Ottawa, Ottawa, ON, Canada; \\ ${ }^{b}$ Toronto General Hospital, University Health Network, Toronto, ON, Canada; ${ }^{\circ}$ King Abdullah \\ Bin Abdulaziz University Hospital, Riyadh, Saudi Arabia; ${ }^{\mathrm{d}}$ Toronto Western Hospital, \\ University Health Network, Toronto, ON, Canada; ${ }^{\mathrm{e}}$ Department of Medicine, University of \\ Toronto, Toronto, ON, Canada; ${ }^{\mathrm{f} D e p a r t m e n t}$ of Speech-Language Pathology, University of \\ Toronto, Toronto, ON, Canada; 9 Toronto Rehabilitation Institute, University Health Network, \\ Toronto, ON, Canada; ${ }^{h}$ Department of Psychiatry and Behavioural Neurosciences, McMaster \\ University, Hamilton, ON, Canada; 'Department of Psychiatry, University of Toronto, Toronto, \\ ON, Canada; ${ }^{j}$ Health Care and Outcomes Research, Krembil Neuroscience Research Institute, \\ Toronto, ON, Canada
}

\section{Keywords}

Ischemic stroke - Magnetic resonance imaging · Aphasia - Dysphagia - Dysarthria ·

Rehabilitation · Clinical predictors

\begin{abstract}
Background: Due to the high post-stroke frequency of dysphagia, dysarthria, and aphasia, we developed comprehensive neuroanatomical, clinical, and demographic models to predict their presence after acute ischemic stroke. Methods: The sample included 160 randomly selected first-ever stroke patients with confirmed infarction on magnetic resonance imaging from 1 tertiary stroke center. We documented acute lesions within 12 neuroanatomical regions and their associated volumes. Further, we identified concomitant chronic brain disease, including atrophy, white matter hyperintensities, and covert strokes. We developed predictive models using logistic regression with odds ratios (OR) and their $95 \%$ confidence intervals (95\% CI) including demographic, clinical, and acute and chronic neuroanatomical factors. Results: Predictors of dysphagia included medullary (OR 6.2, 95\% CI 1.5-25.8), insular (OR 4.8, 95\% CI 2.0-11.8), and pontine (OR 3.6, 95\% CI 1.2-10.1) lesions, followed by brain atrophy (OR 3.0, 95\% CI 1.04-8.6), internal capsular lesions (OR 2.9, 95\% CI 1.2-6.6), and increasing age (OR $1.4,95 \%$ CI 1.1-1.8). Predictors of dysarthria included pontine (OR 7.8, 95\% CI 2.7-22.9), insular (OR 4.5, 95\% CI 1.8-11.4), and internal capsular (OR 3.6, 95\% CI 1.6-7.9) lesions. Predictors
\end{abstract}


of aphasia included left hemisphere insular (OR 34.4, 95\% CI 4.2-283.4), thalamic (OR 6.2, 95\% CI 1.6-24.4), and cortical middle cerebral artery (OR 4.7, 95\% CI 1.5-14.2) lesions. Conclusion: Predicting outcomes following acute stroke is important for treatment decisions. Determining the risk of major post-stroke impairments requires consideration of factors beyond lesion localization. Accordingly, we demonstrated interactions between localized and global brain function for dysphagia and elucidated common lesion locations across 3 debilitating impairments.

(C) 2017 The Author(s)

Published by S. Karger AG, Basel

\section{Introduction}

Dysphagia, dysarthria, and aphasia occur in isolation or concomitantly in two-thirds of all first-ever acute ischemic stroke patients [1]. Dysphagia may result in negative acute-stage outcomes such as an increased length of hospital stay [2] and aspiration pneumonia [3]. Patients with dysphagia are more likely to develop pneumonia than those without [4], whereby the presence of pneumonia incurs higher inpatient mortality rates $[2,5,6]$. In fact, a recent population study demonstrated an in-hospital mortality rate of $20 \%$ for stroke patients with pneumonia compared to $3.5 \%$ for those without [6]. Notwithstanding, dysphagia-related negative outcomes may be partially due to increased stroke severity [7] and/ or a decreased level of alertness [1]. Likewise, communication impairments, whether isolated or co-occurring with dysphagia, are also associated with poor outcomes. Dysarthria incurs negative social and emotional sequelae [8] and often persists for months beyond the acute presentation [9]. Aphasia is associated with negative acute-stage outcomes such as increased length of hospital stay [10] and in-hospital death [10]. Given the high frequency [1], co-occurrence [1], and negative sequelae of dysphagia, dysarthria, and aphasia after ischemic stroke $[3,8,10]$, accurately identifying their presence is necessary to promote early and comprehensive management.

At stroke onset, dysarthria and/or aphasia may be the chief or sole symptomatic manifestations [9], facilitating a rapid diagnosis of stroke even prior to emergency room arrival [11]. Without measures for early detection, dysphagia may be less salient symptomatically than dysarthria and aphasia, thereby compromising timely medical interventions such as alternatives to oral medication [12] and increasing the risk of aspiration pneumonia [13]. Also, given the potential risks of thrombolytic or endovascular therapy for patients with acute ischemic stroke [14], it is important to be able to predict major post-stroke impairments based on initial imaging and clinical and demographic factors. Knowing the key factors associated with the presence of 1 or multiple debilitating impairments may facilitate decisionmaking for medical interventions where potential benefits outweigh risks. That is, patients at high risk of developing long-lasting sequelae and impaired functions would be more likely to have expedient and rapid treatment with time-dependent interventions such as endovascular [15] and/or tissue plasminogen activator [16] therapy.

Usual clinical practice in the diagnosis of ischemic stroke includes the documentation of symptoms and signs relative to stroke onset and evaluation by computerized tomography and/or magnetic resonance (MR) brain imaging. MR imaging (MRI) is particularly valuable in the diagnosis of acute ischemic stroke [17]. In addition to standard T1- and T2-weighted MR sequences, diffusion-weighted imaging (DWI) can confirm infarction within minutes of its onset [18], thereby providing a known time course for potential recovery. Knowing the MR-based predictors of post-stroke impairments such as dysphagia, dysarthria, and aphasia will aid in developing protocols for their early identification and ensuing management. 
To our knowledge, only 3 articles have demonstrated predictive models based on MRI, exclusively for dysphagia [19-21], with effects for pontine and medullary lesions in infratentorial regions [19] and for internal capsular [20,21] and insular cortex lesions [20] in supratentorial regions. Despite attempting to account for demographics [19] and stroke severity $[20,21]$, the authors failed to identify effects apart from acute lesion localization in their models. However, equally important is the development of whole brain predictive models that include demographic, clinical, and chronic as well as acute brain factors relative to the expression of debilitating impairments following stroke. Consequently, our objective was to model demographic, clinical, and whole brain MRI-based acute and chronic neuroanatomical predictors of dysphagia, dysarthria, and aphasia early after first-ever ischemic stroke.

\section{Subjects and Methods}

\section{Patient Selection}

The current investigation involved a large consecutive cohort of acute ischemic stroke patients with MRI from the Ontario Stroke Registry (OSR). In a previous study, we randomly selected 250 patients from the OSR database, conducting a supplementary medical chart review of 221 available patient records at a tertiary care regional stroke center [1]. The study was approved as a medical record review by the institutional ethics board and the procedures were in accordance with institutional guidelines. The 221 patients composed the initial cohort for the present study, further restricted to patients with MR-confirmed acute cerebral infarction within 14 days of stroke onset. We then excluded patients with a previous history of dementia and those with MR evidence of existing nonstroke neurological lesions, such as brain tumors, abscesses, contusions, and previous brain surgery.

\section{Demographic and Clinical Factors}

The procedures and reliability in the detection of dysphagia, dysarthria, and aphasia were consistent with our previous investigation describing their frequency and co-occurrence [1]. Dysphagia identification resulted from clinical or instrumental assessment by speech-language pathologists or radiographic confirmation of enteral feed insertion [1]. Dysarthria and aphasia identification was based on clinical assessment by speech-language pathologists, physicians, or stroke nurses [1]. We extracted demographic (age and sex) and clinical factors (stroke risk factors, comorbidities, and stroke severity) from the OSR database.

\section{MR Sequences}

Standard acute stroke imaging involved a 1.5-T Signa EchoSpeed MR scanner (version 8.2.3; GE Healthcare) with a quadrature head coil. The imaging protocol included a sagittal T1-weighted sequence (TR/TE $450 / 20 \mathrm{~ms}$ ) with a $7.5-\mathrm{mm}$ section thickness and 2-mm spacing, an axial T2-weighted FLAIR (fluid-attenuated inversion recovery) sequence (TR/TE 9,000/165 ms; TI 2,200 ms) with a 5-mm section thickness and 2-mm spacing, and an isotropic axial diffusion sequence $\left(B=1,000 \mathrm{~s} / \mathrm{mm}^{2}\right.$; TR/TE $\left.11,000 / 59 \mathrm{~ms}\right)$ with a 5 -mm section thickness and 0-mm spacing.

\section{Acute Lesion Variables}

For lesion localization, we identified 12 regions of interest (ROIs) from the previous literature involving post-stroke MRI correlates of dysphagia [19-21], dysarthria [9], and aphasia [22]. We selected the first positive acute DWI scan, documenting all lesion projections onto affected ROIs and their laterality using a whole brain template (Fig. 1). For aphasia, we restricted analyses to left hemisphere or bilateral involvement. One rater (H.L.F.) manually 


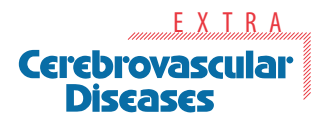

ebrovascular
Diseases

\begin{tabular}{l|l}
\hline Cerebrovasc Dis Extra \\
\hline DOI: $10.1159 / 000457810$ & $\begin{array}{l}\text { (c) 2017 The Author(s). Published by S. Karger AG, Basel } \\
\text { www.karger.com/cee }\end{array}$ \\
\hline
\end{tabular}

Flowers et al:: MRI-Based Neuroanatomical Predictors of Dysphagia, Dysarthria, and Aphasia in Patients with First Acute Ischemic Stroke

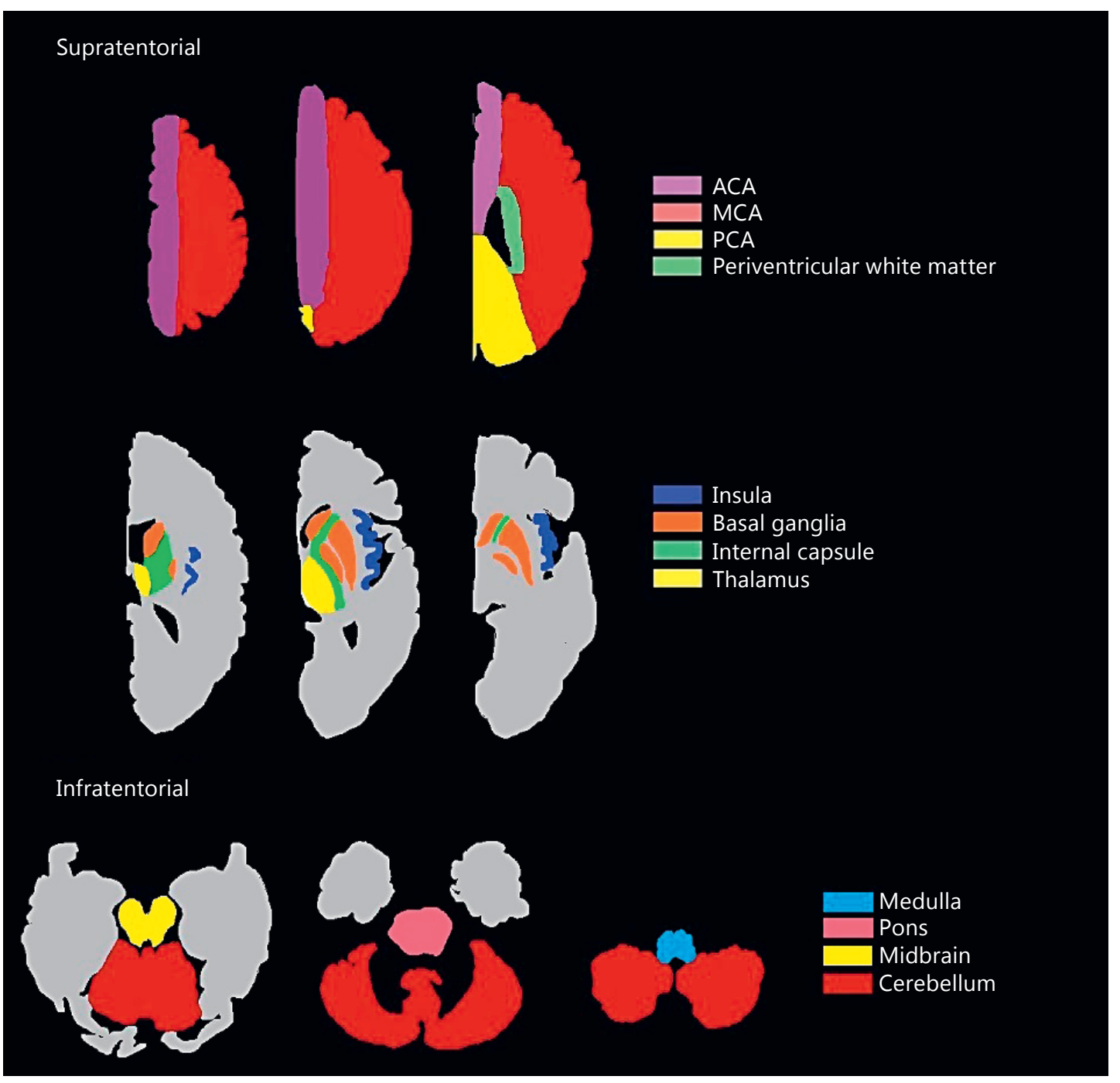

Fig. 1. Regions of interest throughout the brain based on the ch2better template in MRIcron [23]. ACA, anterior cerebral artery; MCA, middle cerebral artery; PCA, posterior cerebral artery.

traced the lesions on each DWI slice with MRIcron [23] and calculated their volumes in cubic centimeters multiplied by 5 to account for slice thickness.

\section{Chronic Neurological Disease Variables}

The FLAIR image, along with any available MR and/or computed tomography modalities, served in identifying covert stroke, brain atrophy, and white matter hyperintensities. We classified covert strokes [24] into 4 categories: (1) a single lesion 10-20 $\mathrm{mm}$ in the longest diameter; (2) multiple lesions 10-20 mm in the longest diameter; (3) a single lesion $>20 \mathrm{~mm}$ in the longest diameter; and (4) multiple lesions, with at least 1 of them $>20 \mathrm{~mm}$ in the longest diameter. We rated brain atrophy as none, mild, moderate, or severe, from overall subjective determination of cortical contraction [25], lateral ventricular enlargement [26], Sylvian fissure enlargement [27], and/or localized gray tissue atrophy [28]. For white matter disease, we combined Fazekas scale [29] ratings of periventricular (PV) hyperintensities and deep white matter (DWM) hyperintensities. Scores of 0 indicated no PV or DWM hyperintensities, 
Fig. 2. Flow diagram of eligible patients from the Ontario Stroke Registry database (July 1, 2003, to March 31, 2008). MRI, magnetic resonance imaging; DWI, diffusion-weighted imaging.

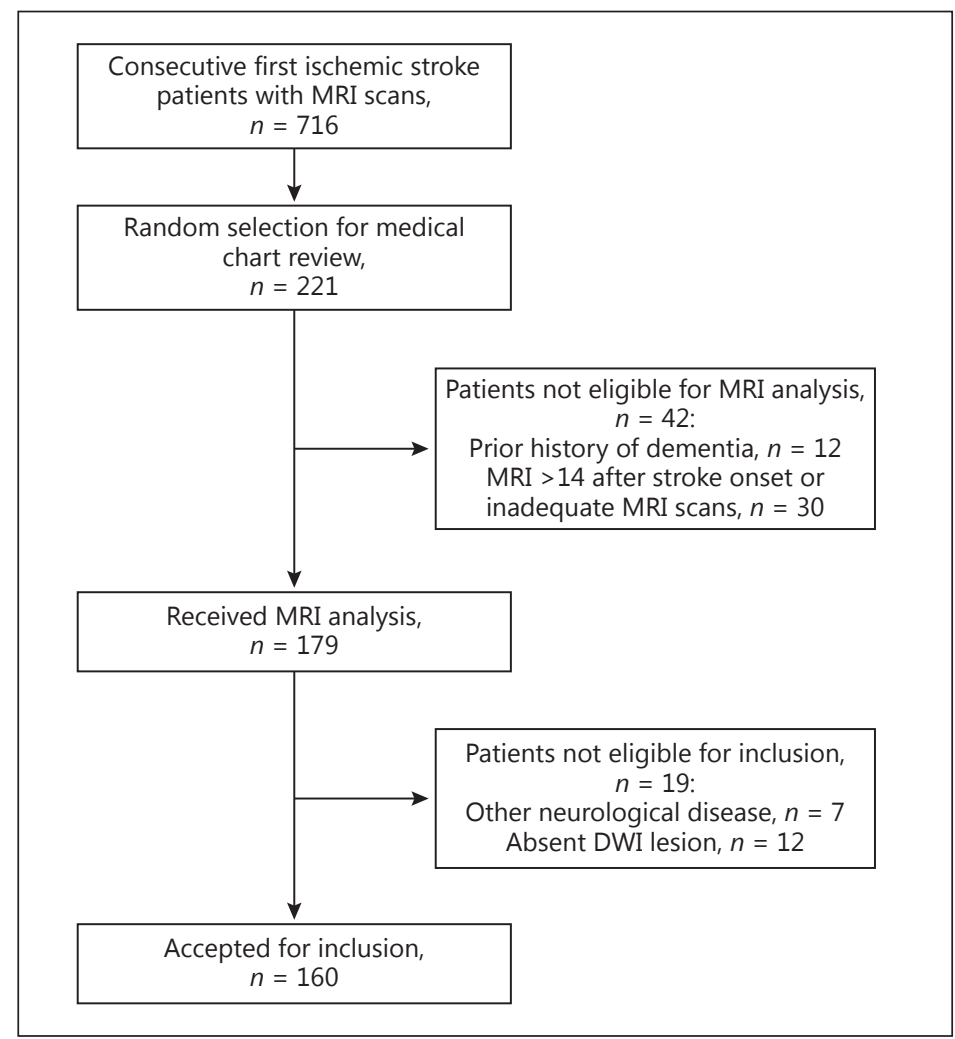

1 scattered PV and/or DWM hyperintensities, 2 semiconfluent PV and/or DWM hyperintensities, and 3 confluent and extensive PV and/or DWM hyperintensities.

\section{MRI Scan Consensus}

A trained rater (H.L.F.) and a neuroradiologist (M.A.A.) independently evaluated all MRI scans, remaining blind to clinical presentation, including dysphagia, dysarthria, and/or aphasia status. They documented the chronic brain disease and acute lesion attributes for all patients. Subsequently, the 2 raters and a senior neuroradiologist (D.M.) and/or senior neurologist (F.L.S.) resolved any discrepant items by consensus.

\section{Statistical Analysis}

For descriptive tabulations of the data, we suppressed total numbers of patients $\leq 10$ (so that small cells of $n \leq 5$ could not potentially be recomputed) according to privacy policies at the Institute for Clinical and Evaluative Sciences, which houses the OSR. We conducted multivariable logistic regression modeling to evaluate predictors of dysphagia, dysarthria, and aphasia, using a backward selection model with a $p<0.05$ cutoff, to obtain odds ratios (OR) and their 95\% confidence intervals (95\% CI). Logistic regression modelling enabled us to simultaneously evaluate demographic, clinical, and neuroanatomical factors in a single model according to impairment status. Given neighboring affected ROIs in the models, we also computed variance inflation factors (VIFs) by reanalyzing the models with linear regression and associated collinearity diagnostics. We considered potential multicollinearity where the VIF was $>6$ [30]. We tested our logistic regression models for predictive accuracy using Nagelkerke's $R^{2}$, and for discriminative probability using the concordance (c) statistic for each impairment. 
Cerebirovascular
Diseases
Cerebrovasc Dis Extra

DOI: $10.1159 / 000457810$

(C) 2017 The Author(s). Published by S. Karger AG, Basel www.karger.com/cee

Flowers et al.: MRI-Based Neuroanatomical Predictors of Dysphagia, Dysarthria, and Aphasia in Patients with First Acute Ischemic Stroke

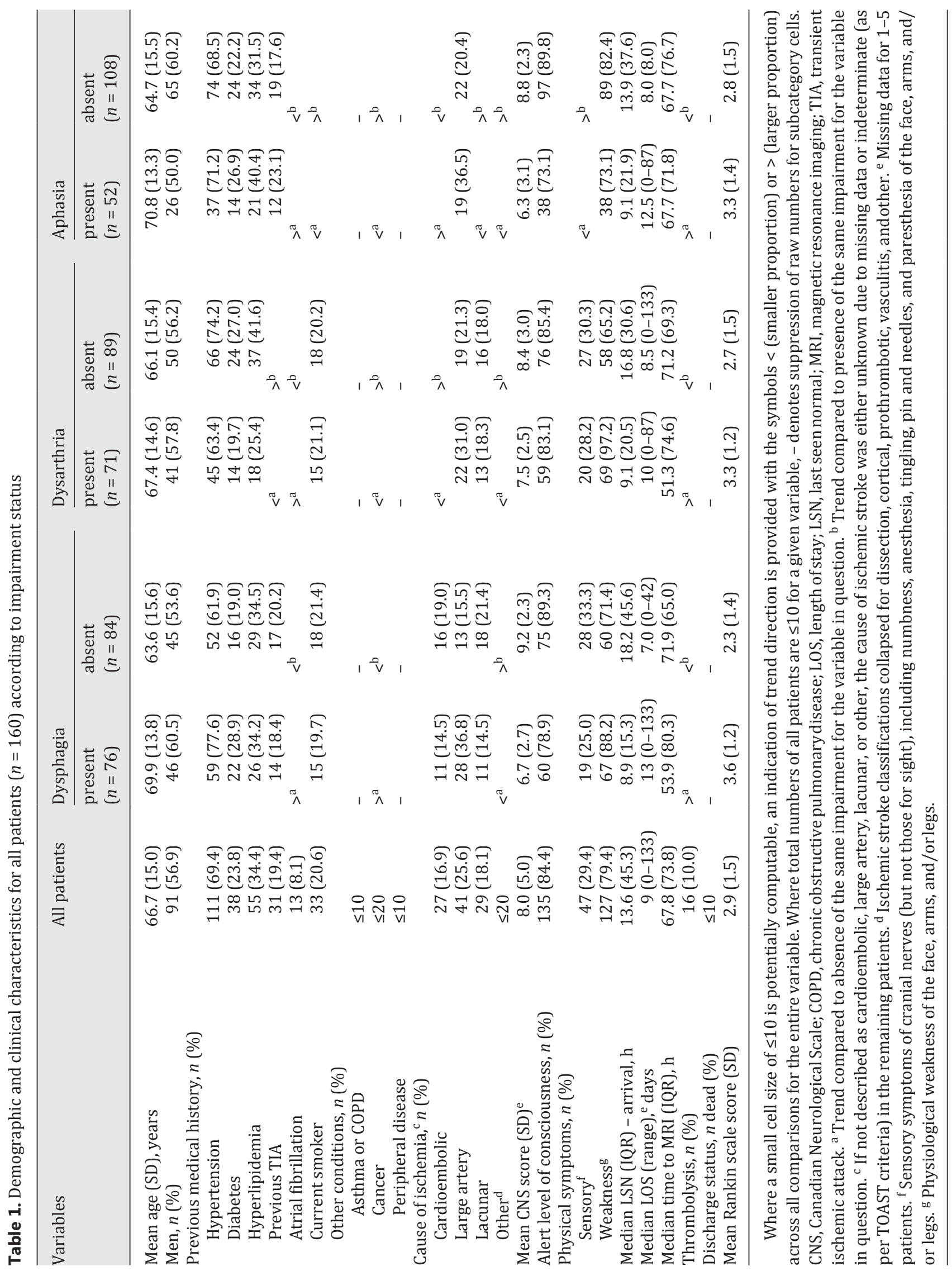


Flowers et al.: MRI-Based Neuroanatomical Predictors of Dysphagia, Dysarthria, and Aphasia in Patients with First Acute Ischemic Stroke

\section{Results}

One hundred and sixty of the 221 patients [1] met our inclusion criteria for MRI scan review (Fig. 2). They had a mean age of 68 years, and $57 \%$ were men (Table 1). Dysphagia, dysarthria, and aphasia were present in 76 (48\%), 71 (44\%), and 52 (33\%) of the patients, respectively (Table 2). Sixteen patients (10\%) had all 3 impairments, while $110(69 \%)$ had at least 1 of the 3 impairments. Dysphagia and dysarthria co-occurred in 51 (32\%), dysphagia and aphasia in 29 (18\%), and dysarthria and aphasia in $25(16 \%)$ of the patients.

\section{MRI Characteristics}

There was at least mild brain atrophy in $45 \%$ of the patients, whereas white matter hyperintensities occurred in $94 \%$ and prior covert stroke in $30 \%$ (Table 2). The median acute lesion volume was $23 \mathrm{~cm}^{3}$ (Q1 5.5, Q3 78.2) (Table 2). One hundred and seventeen (73\%) of the patients had isolated supratentorial lesions, $32(20 \%)$ had isolated infratentorial lesions, and $11(7 \%)$ had mixed lesions (supra- and infratentorial). Additionally, the 16 patients with all 3 impairments had supratentorial involvement.

\section{Predictive Models}

The multivariable logistic regression analyses for demographic, clinical, and whole brain neuroanatomical predictors of dysarthria and dysphagia revealed the highest effects for pontine (OR 7.8, 95\% CI 2.7-22.9) and medullary (OR 6.2, 95\% CI 1.5-25.8) lesions, respectively (Table 3). The analyses for aphasia (excluding patients with right-sided lesions, $n=$ 102) revealed the highest effect for insular lesions (OR 34.4, 95\% CI 4.2-283.4) (Table 3). The highest effects in patients with isolated supratentorial lesions $(n=117)$ included insular lesions for aphasia (OR 10.5, 95\% CI 1.2-94.0), at least moderate brain atrophy for dysphagia (OR 6.2, 95\% CI 1.8-21.1), and insular lesions for dysarthria (OR 5.0, 95\% CI 1.8-13.4) (Table 4). All VIFs were below the preestablished threshold indicating absence of extreme multicollinearity. The Nagelkerke $R^{2}$ values for the whole brain and supratentorial models were 0.28 and 0.36 for dysphagia, 0.23 and 0.22 for dysarthria, and 0.48 and 0.52 for aphasia, respectively. The corresponding c statistics for the whole brain and supratentorial models were 0.76 and 0.80 for dysphagia, 0.75 and 0.74 for dysarthria, and 0.83 and 0.90 for aphasia.

\section{Discussion}

We demonstrated whole brain neuroanatomical predictors of dysphagia, dysarthria, and aphasia after first-ever acute ischemic stroke, with models inclusive of demographic, clinical, and acute and chronic neuroanatomical factors. As expected, acute lesion factors were the strongest predictors of all 3 impairments, yet with concomitant contributions of brain atrophy and increasing age for dysphagia. All 3 impairments showed high effects for insular involvement, suggesting its critical role in integrative function across motor and/or higher cognitive behaviors [31].

Acute stroke ROIs predictive of dysphagia involved an extensive neuroanatomical substrate, confirming the relationship between post-stroke dysphagia and pontine [19], medullary [19], internal capsular [20,21], and insular [20] involvement. Additionally, we demonstrated critical factors beyond lesion location for dysphagia, including age and at least moderate premorbid brain atrophy. Swallowing involves integration of volitional and reflexive behaviors across motor and sensory modalities. Consequently, insults to both highly localized and globally mediated neuroanatomical regions may incur a high risk of post-stroke dysphagia. That is, the manifestation of dysphagia may represent influences of prior loss in 
Flowers et al.: MRI-Based Neuroanatomical Predictors of Dysphagia, Dysarthria, and Aphasia in Patients with First Acute Ischemic Stroke

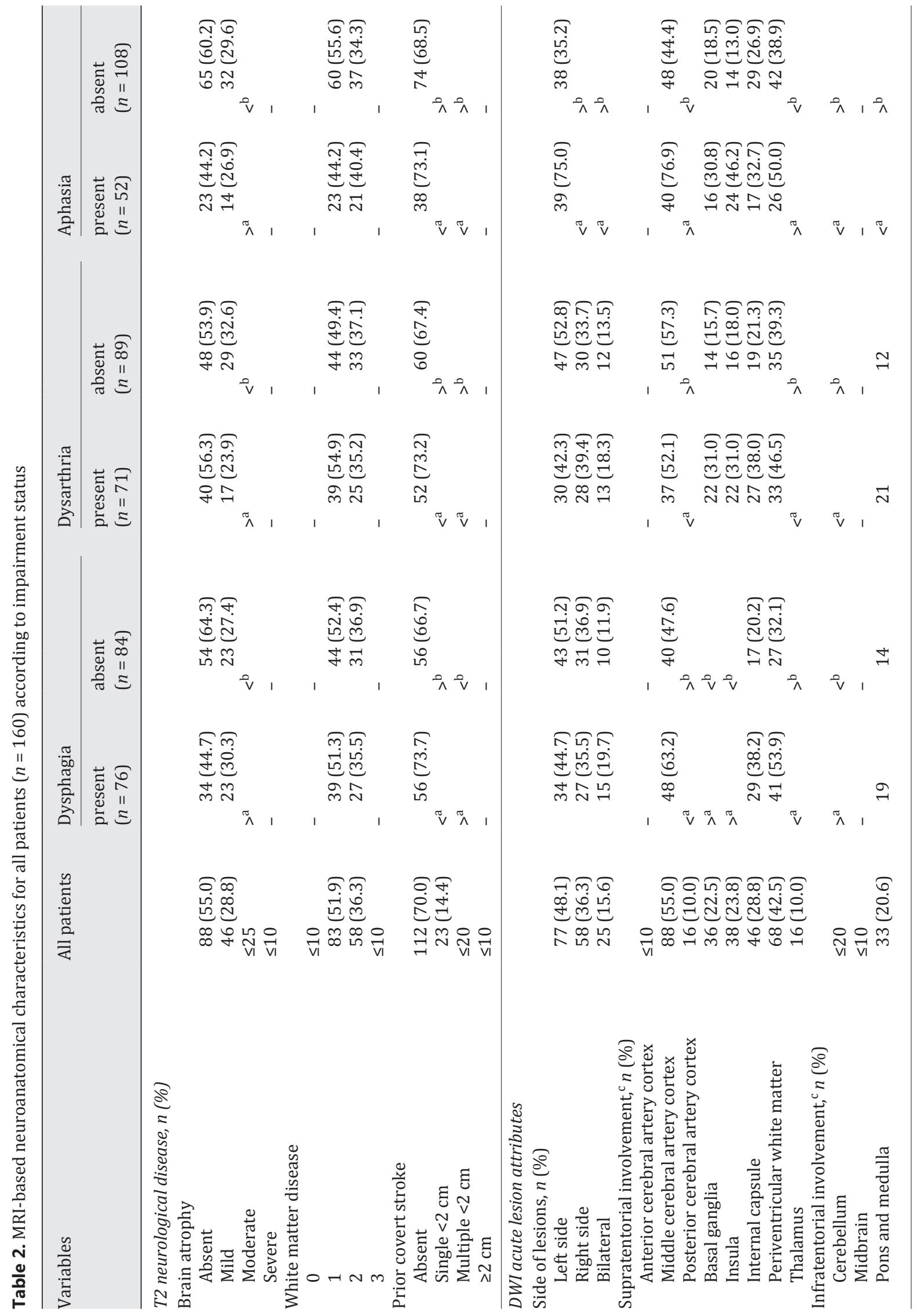


Flowers et al.: MRI-Based Neuroanatomical Predictors of Dysphagia, Dysarthria, and Aphasia in Patients with First Acute Ischemic Stroke

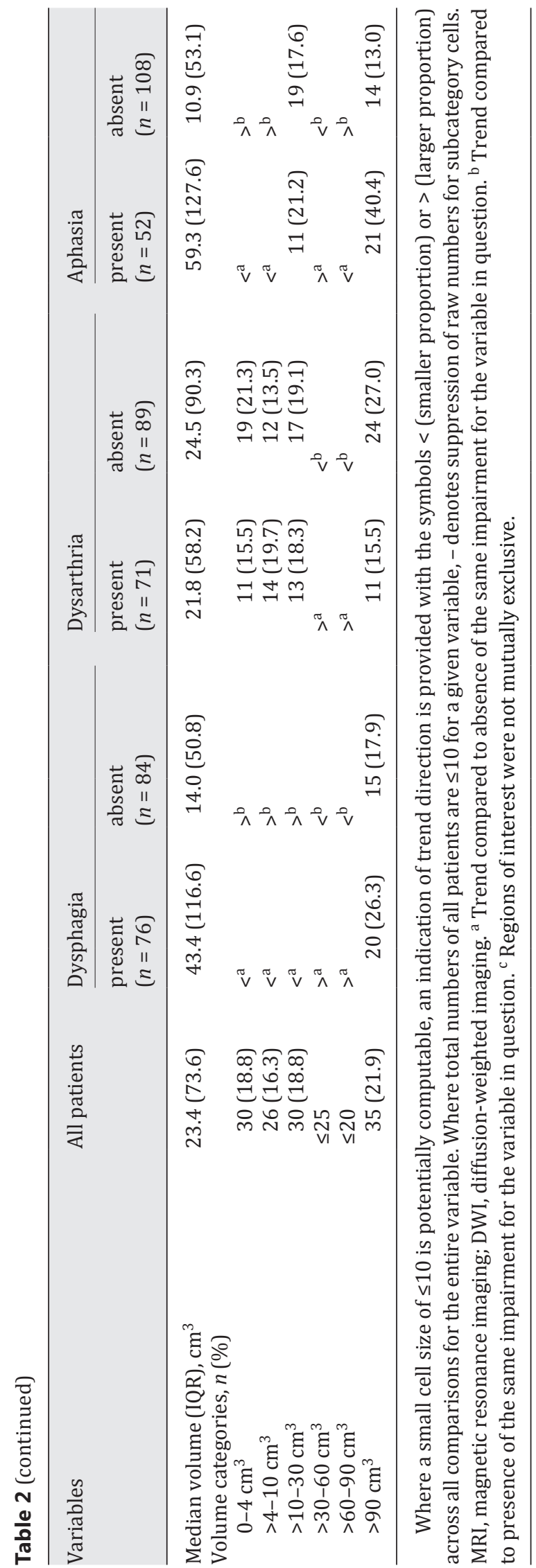



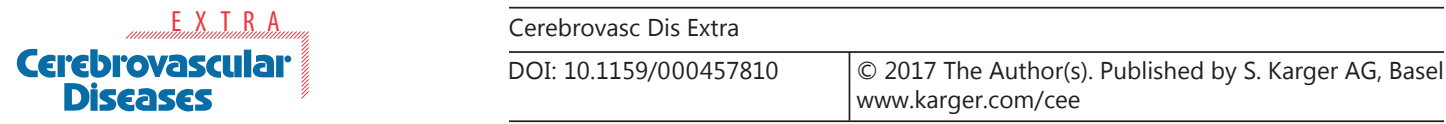

Flowers et al.: MRI-Based Neuroanatomical Predictors of Dysphagia, Dysarthria, and Aphasia in Patients with First Acute Ischemic Stroke

Table 3. Whole brain neuroanatomical and demographic predictors of dysphagia, dysarthria, and aphasia

\begin{tabular}{|c|c|c|c|}
\hline Predictor variables & $\begin{array}{l}\text { Dysphagia, OR }(95 \% \mathrm{CI}) \\
(n=76)\end{array}$ & $\begin{array}{l}\text { Dysarthria, OR }(95 \% \mathrm{CI}) \\
(n=71)\end{array}$ & $\begin{array}{l}\text { Aphasia, OR }(95 \% \mathrm{CI}) \\
(n=49)^{\mathrm{a}}\end{array}$ \\
\hline \multicolumn{4}{|l|}{ Demographic } \\
\hline Age (10-year increments) & $1.4(1.1-1.8)$ & eliminated & eliminated \\
\hline Sex (female) & eliminated & eliminated & eliminated \\
\hline \multicolumn{4}{|l|}{ Clinical } \\
\hline History of atrial fibrillation (yes) & eliminated & eliminated & eliminated \\
\hline \multicolumn{4}{|l|}{ Chronic brain disease } \\
\hline Brain atrophy (moderate or severe) & $3.0(1.04-8.6)$ & eliminated & eliminated \\
\hline White matter hyperintensities (scores $\geq 2$ ) & eliminated & eliminated & eliminated \\
\hline Covert stroke (multiple or large) & eliminated & eliminated & eliminated \\
\hline \multicolumn{4}{|l|}{ Neuroanatomical regions of interest } \\
\hline Medulla (yes) & $6.2(1.5-25.8)$ & eliminated & not tested \\
\hline Pons (yes) & $3.6(1.2-10.1)$ & $7.8(2.7-22.9)$ & not tested \\
\hline Cerebellum (yes) & eliminated & eliminated & not tested \\
\hline Thalamus (yes) & eliminated & not tested & $6.2(1.6-24.4)$ \\
\hline Basal ganglia (yes) & eliminated & eliminated & eliminated \\
\hline Internal capsule (yes) & $2.9(1.2-6.6)$ & $3.6(1.6-7.9)$ & eliminated \\
\hline Insula (yes) & $4.8(2.0-11.8)$ & $4.5(1.8-11.4)$ & $34.4(4.2-283.4)$ \\
\hline Periventricular white matter (yes) & eliminated & eliminated & eliminated \\
\hline Anterior cerebral artery (yes) & eliminated & eliminated & eliminated \\
\hline Middle cerebral artery (yes) & eliminated & eliminated & $4.7(1.5-14.2)$ \\
\hline Posterior cerebral artery (yes) & eliminated & eliminated & eliminated \\
\hline Lesion volume (increasing per mL) & eliminated & $0.997(0.995-1.000)$ & eliminated \\
\hline Lesion laterality (right) & eliminated & eliminated & not tested \\
\hline
\end{tabular}

Table 4. Supratentorial predictors of dysphagia, dysarthria, and aphasia

\begin{tabular}{|c|c|c|c|}
\hline Independent variables & $\begin{array}{l}\text { Dysphagia, OR }(95 \% \mathrm{CI}) \\
(n=54)\end{array}$ & $\begin{array}{l}\text { Dysarthria, OR }(95 \% \mathrm{CI}) \\
(n=49)\end{array}$ & $\begin{array}{l}\text { Aphasia, OR }(95 \% \mathrm{CI}) \\
(n=44)^{\mathrm{a}}\end{array}$ \\
\hline \multicolumn{4}{|l|}{ Demographic } \\
\hline Age (10-year increments) & eliminated & eliminated & eliminated \\
\hline Sex (female) & eliminated & eliminated & eliminated \\
\hline \multicolumn{4}{|l|}{ Clinical } \\
\hline History of atrial fibrillation (yes) & eliminated & eliminated & eliminated \\
\hline \multicolumn{4}{|l|}{ Chronic brain disease } \\
\hline Brain atrophy (moderate or severe) & $6.2(1.8-21.1)$ & eliminated & eliminated \\
\hline White matter hyperintensities (score $\geq 2$ ) & eliminated & eliminated & eliminated \\
\hline Covert stroke (multiple or large) & eliminated & eliminated & eliminated \\
\hline \multicolumn{4}{|l|}{ Neuroanatomical regions of interest } \\
\hline Thalamus (yes) & $0.07(0.01-0.8)$ & not tested & eliminated \\
\hline Basal ganglia (yes) & eliminated & eliminated & eliminated \\
\hline Internal capsule (yes) & $3.0(1.3-7.3)$ & $4.0(1.7-9.2)$ & eliminated \\
\hline Insula (yes) & $3.9(1.6-10.0)$ & $5.0(1.8-13.4)$ & $10.5(1.2-94.0)$ \\
\hline Periventricular white matter (yes) & eliminated & eliminated & eliminated \\
\hline Anterior cerebral artery (yes) & eliminated & eliminated & eliminated \\
\hline Middle cerebral artery (yes) & eliminated & eliminated & eliminated \\
\hline Posterior cerebral artery (yes) & eliminated & eliminated & eliminated \\
\hline Lesion volume (increasing per mL) & eliminated & $0.997(0.995-1.000)$ & $1.03(1.01-1.05)$ \\
\hline Lesion laterality (right) & eliminated & eliminated & not tested \\
\hline
\end{tabular}


Flowers et al.: MRI-Based Neuroanatomical Predictors of Dysphagia, Dysarthria, and Aphasia in Patients with First Acute Ischemic Stroke

tertiary mediation combined with acute stroke lesions, thereby grossly compromising the global and local pathways necessary for sensorimotor integration.

Lesioned neuroanatomical regions predictive of dysarthria were also represented throughout the brain. We confirmed the relationship between pontine [9, 32], internal capsular [32], and insular [32] lesions and dysarthria. Future research is necessary to investigate more discrete lesion locations relative to dysarthria subtypes, and to elucidate neuroanatomical underpinnings for co-occurring dysarthria and dysphagia.

Lesioned neuroanatomical regions predictive of left hemisphere aphasia suggested cortical and subcortical integrative mechanisms. Our findings support a previous study involving a large cohort of MR-confirmed acute stroke patients [33]. Neuroanatomical regions associated with post-stroke aphasia included inferior frontal and insular involvement for Broca's aphasia, insular and temporoparietal involvement for Wernicke's aphasia, and large predominantly cortical frontal to posterior temporal involvement for global aphasia [33]. Moreover, after isolated subcortical strokes, patients with aphasia had thalamic involvement more frequently than those without [33]. Our whole brain predictive model of aphasia reflects similar gross neuroanatomical trends, given effects for lesions to superficial middle cerebral artery, insular, and thalamic regions. Continued research is necessary to investigate lesions within discretely localized regions, such as the temporal pole [34] and dorsal or ventral white matter paths [35], relative to specific language operations [34, 35]. We further suggest a delineation of insular involvement to develop an understanding of its function relative to isolated or co-occurring impairments. We may consequently ameliorate early management protocols and/or cross-system interventions targeting multiple impairments [36] early after stroke onset. Currently, our findings highlight the need for continued consideration of age, chronic brain disease, and lesion volume in the determination of risk of impairment and brain-behavior relationships.

The potential clinical application of our findings include careful attention to patients presenting with signs of left middle cerebral artery stroke (and likely insular involvement), given the high risk of both motor and higher cognitive impairments. Similarly, patients with stroke suggestive of medullary and/or pontine involvement should have early vigilant attention in the management of swallowing and speech. Whether or not there is access to MRI at a given institution, early screening for swallowing and communication with rapid referral to speech-language pathology for patients who fail screening should constitute the standard of care.

By extension, our findings provide an impetus for the continued use and development of rapid and accurate screening tools [37-39] to establish their concordance with stroke severity scales such as the National Institutes of Health Stroke Scale (NIHSS) [40]. The NIHSS is appropriate for identifying stroke severity, but extracted subscores may not be suitable for screening (e.g., for language or other domains), since single subtests may have poor reliability [41]. Consequently, separate rapid screening tools for swallowing and communication are highly advisable. To ensure monitoring of suspected rapid recovery or deterioration, repeat testing with validated rapid screening tools is essential [42].

Similarly, rapid assessment tools validated according to MRI indicators of impairment could provide surrogate information about stroke severity, volume, and/or lesion location. Consequently, such screening and assessment tools could permit the most accurate prognosis [20] and comprehensive management with a comparable standard of care in centers with and without MRI. Where possible, we advocate the use of MRI in ischemic stroke patients, as it remains the gold standard for identifying the presence and location of acute cerebral infarction. Computed tomography may fail to identify early infarction particularly within the brainstem or cerebellum $[17,43]$. Continued clinical initiatives and research with MRI have the capacity to further inform practice. They will aid in establishing neuroanatomical prognostic indicators 
of recovery or indicators of continued risk and will inform therapeutic goal-setting, especially where a given intervention may confer distributed effects across multiple impairments [36].

Despite our inclusion of a large homogeneous sample of first-ever stroke patients with MR-confirmed infarction, our study has limitations. First, our retrospective design potentially led to restricted detection of patients with mild impairments or those difficult to assess. Second, we included only patients with MRI within 2 weeks of stroke onset. Older patients, those with multiple comorbidities (potentially also requiring intensive medical management), and those with severe strokes may not undergo MRI early after stroke onset. Finally, our broadly specified cortical regions did not permit a close exploration of neuroanatomical areas that might be particularly important in discrete functions, such as volitional control of swallowing, motor initiation of speech, and the localization and/or integration of receptive and expressive language. However, we have initially employed comprehensive models inclusive of factors beyond acute lesion location.

\section{Conclusion}

We provided comprehensive and clinically motivated models for predicting dysphagia, dysarthria, and aphasia in a homogeneous sample of first-ever ischemic stroke patients. We have shown that the neuroanatomical substrate for dysphagia is locally represented throughout the brain but further compromised by premorbid deficits in brain integrity. We confirmed discretely localized neuroanatomical substrates for dysarthria and aphasia with concomitant but weak effects for lesion volume. Future studies are necessary to prospectively evaluate physiologic and/or cognitive-linguistic domains of these 3 impairments to derive neuroanatomical predictive models relative to their severity and co-occurrence patterns. We shed new light on interactions between local and global brain function for dysphagia, found expected neuroanatomical predictors of dysarthria and aphasia, and identified common brain regions in the expression of multiple impairments.

\section{Acknowledgments}

The OSR receives an operating grant from the Ontario Ministry of Health and Long-Term Care. The results and conclusions of the current study should be attributed to the authors and not to the funding organizations. H.L.F. received funding from the Heart and Stroke Foundation of Ontario and the Queen Elizabeth II Scholarships in Science and Technology. F.L.S. received salary support through the Canadian Stroke Network. R.M. received a research grant from the Canadian Stroke Network and holds a Canadian Research Chair (Tier II) in Swallowing Disorders.

\section{Disclosure Statement}

The authors have no conflicts of interest to declare.

\section{Author Contributions}

The authors exclusively designed and conducted the study, including data collection, analysis, and interpretations. 


\begin{tabular}{l}
\hline Cerebrovasc Dis Extra \\
\hline DOI: 10.1159/000457810 \\
$\begin{array}{l}\text { (c) 2017 The Author(s). Published by S. Karger AG, Basel } \\
\text { www.karger.com/cee }\end{array}$
\end{tabular}

Flowers et al.: MRI-Based Neuroanatomical Predictors of Dysphagia, Dysarthria, and Aphasia in Patients with First Acute Ischemic Stroke

\section{References}

1 Flowers HL, Silver FL, Fang J, Rochon E, Martino R: The incidence, co-occurrence, and predictors of dysphagia, dysarthria, and aphasia after first-ever acute ischemic stroke. J Commun Disord 2013;46:238-248.

-2 Guyomard V, Fulcher RA, Redmayne O, Metcalf AK, Potter JF, Myint PK: Effect of dysphasia and dysphagia on inpatient mortality and hospital length of stay: a database study. Am Geriatr Soc 2009;57:2101-2106.

-3 Martino R, Foley N, Bhogal S, Diamant N, Speechley M, Teasell R: Dysphagia after stroke: incidence, diagnosis, and pulmonary complications. Stroke 2005;36:2756-2763.

4 Langdon CP, Lee AH, Binns CW: Dysphagia in acute ischaemic stroke: severity, recovery and relationship to stroke type. J Clin Neurosci 2007;14:630-634.

5 Hannawi Y, Hannawi B, Rao CPV, Suarez JI, Bershad EM: Stroke-associated pneumonia: major advances and obstacles. Cerebrovasc Dis 2013;35:430-443.

6 Wilson RD: Mortality and cost of pneumonia after stroke for different risk groups. Stroke Cerebrovasc Dis 2012;21:61-67.

7 Smithard DG, Smeeton NC, Wolfe CDA: Long-term outcome after stroke: does dysphagia matter? Age Ageing 2007;36:90-94.

$>8$ Dickson S, Barbour RS, Brady M, Clark AM, Paton G: Patients' experiences of disruptions associated with poststroke dysarthria. Int J Lang Commun Disord 2008;43:135-153.

9 Canbaz DH, Çelebısoy M, Ozdemırkiran T, Tokucoglu F: Dysarthria in acute ischemic stroke: localization and prognosis. J Neurol Sci 2010;27:20-27.

10 Flowers HL, Skoretz SA, Silver FL, Rochon E, Fang J, Flamand-Roze C, Martino R: Poststroke aphasia frequency, recovery, and outcomes: a systematic review and meta-analysis. Arch Phys Med Rehabil 2016;97:2188-2201.e8.

-11 Krebes S, Ebinger M, Baumann AM, Kellner PA, Rozanski M, Doepp F, Sobesky J, Gensecke T, Leidel BA, Malzahn U, Wellwood I, Heuschmann PU, Audebert HJ: Development and validation of a dispatcher identification algorithm for stroke emergencies. Stroke 2012;43:776-781.

12 Kelly J, Wright D, Wood J: Medicine administration errors in patients with dysphagia in secondary care: a multi-centre observational study. J Adv Nurs 2011;67:2615-2627.

13 Hinchey JA, Shephard T, Furie K, Smith D, Wang D, Tonn S: Formal dysphagia screening protocols prevent pneumonia. Stroke 2005;36:1972-1976.

14 Touma L, Filion KB, Sterling LH, Atallah R, Windle SB, Eisenberg MJ: Stent retrievers for the treatment of acute ischemic stroke: a systematic review and meta-analysis of randomized clinical trials. JAMA Neurol 2016;73: 275-281.

15 Goyal AM, Demchuk BK, Menon ME, Eesa M, Rempel JL, Thornton J, et al: Randomized assessment of rapid endovascular treatment of ischemic stroke. N Engl J Med 2015;372:1019-1030.

-16 Chapman SN, Mehndiratta P, Johansen MC, McMurry TL, Johnston KC, Southerland AM: Current perspectives on the use of intravenous recombinant tissue plasminogen activator (tPA) for treatment of acute ischemic stroke. Vasc Health Risk Manag 2014;10:75-87.

-17 Moreau F, Asdaghi N, Modi J, Goyal M, Coutts SB: Magnetic resonance imaging versus computed tomography in transient ischemic attack and minor stroke: the more you see the more you know. Cerbrovasc Dis Extra 2013;3:130-136.

18 Merino JG, Warach S: Imaging of acute stroke. Nat Neurol 2010;6:560-571.

19 Flowers HL, Skoretz SA, Streiner DL, Silver FL, Martino R: MRI-based neuroanatomical predictors of dysphagia after acute ischemic stroke: a systematic review and meta-analysis. Cerebrovasc Dis 2011;32:1-10.

20 Galovic M, Leisi N, Müller M, Weber J, Abela E, Kägi G, Weder B: Lesion location predicts transient and extended risk of aspiration after supratentorial ischemic stroke. Stroke 2013;44:2760-2767.

21 Gonzalez-Fernandez M, Kleinman JT, Ky PK, Palmer JB, Hillis AE: Supratentorial regions of acute ischemia associated with clinically important swallowing disorders: a pilot study. Stroke 2008;39:3022-3028.

$\checkmark 22$ Price CJ, Seghier ML, Leff AP: Predicting language outcome and recovery after stroke: the PLORAS system. Nat Rev Neurol 2010;6:202-210.

23 Rorden C, Karnath H-O, Bonilha L: Improving lesion-symptom mapping. J Cogn Neurosci 2007;19:1081-1088.

-24 Potter GM, Marlborough FJ, Wardlaw JM: Wide variation in definition, detection, and description of lacunar lesions on imaging. Stroke 2011;42:359-366.

25 Sluimer JD, van der Flier WM, Karas GB, Fox NC, Scheltens P, Barkhof F, Vrenken H: Whole-brain atrophy rate and cognitive decline: longitudinal MR study of memory clinic patients. Radiology 2008;248:590-598.

26 Frisoni GB, Fox NC, Jack CR Jr, Scheltens P, Thompson PM: The clinical use of structural MRI in Alzheimer disease. Nat Rev Neurol 2010;6:67-77.

27 Förstl H, Zerfass R, Geiger-Kabisch C, Sattel H, Besthorn C, Hentschel F: Brain atrophy in normal ageing and Alzheimer's disease. Volumetric discrimination and clinical correlations. Br J Psychiatry 1995;167:739-746.

28 Fjell AM, Walhovd KB, Fennema-Notestine C, McEvoy LK, Hagler DJ, Holland D, Brewer JB, Dale AM: One year brain atrophy evident in healthy aging. J Neurosci 2009;29:15223-15231.

29 Fazekas F, Chawluk JB, Alavi A, Hurtig HI, Zimmerman RA: MR signal abnormalities at 1.5 T in Alzheimer's dementia and normal aging. AJR Am J Roentgenol 1987;149:351-356.

30 Norman GR, Streiner DL: Biostatistics: The Bare Essentials, ed 2. Hamilton, BC Decker, 2010.

-31 Uddin LQ: Salience processing and insular cortical function and dysfunction. Nat Rev Neurosci 2015;16: $55-61$. 
-32 Kumral E, Çelebısoy M, Çelebısoy N, Canbaz DH, Çallı C: Dysarthria due to supratentorial and infratentorial ischemic stroke: a diffusion-weighted imaging study. Cerebrovasc Dis 2007;23:331-338.

33 Kreisler A, Godefroy O, Delmaire C, Debachy B, Leclercq M, Pruvo JP, Leys D: The anatomy of aphasia revisited. Neurology 2000;54:1117-1123.

-34 Magnusdottir S, Fillmore P, den Ouden DB, Hjaltason H, Rorden C, Kjartansson O, Bonilha L, Fridriksson J: Damage to left anterior temporal cortex predicts impairment of complex syntactic processing: a lesionsymptom mapping study. Hum Brain Mapp 2013;34:2715-2723.

-35 Kümmerer D, Hartwigsen G, Kellmeyer P, Glauche V, Mader I, Klöppel S, Suchan J, Karnath HO, Weiller C, Saur D: Damage to ventral and dorsal language pathways in acute aphasia. Brain 2013;136(pt 2):619-629.

-36 McFarland DH, Tremblay P: Clinical implications of cross-system interactions. Semin Speech Lang 2006;27: 300-309.

37 El Hachioui H, Visch-Brink EG, de Lau LM, van de Sandt-Koenderman MW, Nouwens F, Koudstaal PJ, Dippel DW: Screening tests for aphasia in patients with stroke: a systematic review. J Neurol 2017;264:211-220.

-38 Schepp SK, Tirschwell DL, Miller RM, Longstreth WT Jr: Swallow screens after stroke: a systematic review. Stroke 2012;43:869-871.

-39 Martino R, Flowers HL, Shaw SM, Diamant NE: A systematic review of current clinical and instrumental swallowing assessment methods. Curr Phys Med Rehabil Rep 2013;1:267-279.

40 Brott T, Adams HP Jr, Olinger CP, Marler JR, Barsan WG, Biller J, et al: Measurements of acute cerebral infarction: a clinical examination scale. Stroke 1989;20:864-870.

41 Meyer BC, Lyden PD: The modified National Institutes of Health Stroke Scale (mNIHSS): its time has come. Int J Stroke 2009;4:267-273.

-42 Flamand-Roze C, Cauquil-Michon C, Denier C: Tools and early management of language and swallowing disorders in acute stroke patients. Curr Neurol Neurosci Rep 2012;12:34-41.

-43 Lövblad KO, Laubach HJ, Baird AE, Curtin F, Schlaug G, Edelman RR, Warach S: Clinical experience with diffusion-weighted MR in patients with acute stroke. AJNR Am J Neuroradiol 1998;19:1061-1066. 